\title{
Hydrodynamic type integrable equations on a segment and a half-line
}

\author{
Metin Gürses, ${ }^{1, a)}$ Ismagil Habibullin, ${ }^{1, b)}$ and Kostyantyn Zheltukhin ${ }^{2, c)}$ \\ ${ }^{1}$ Department of Mathematics, Faculty of Sciences, Bilkent University, \\ 06800 Ankara, Turkey \\ ${ }^{2}$ Department of Mathematics, Faculty of Sciences, Middle East Technical University, \\ 06531 Ankara, Turkey
}

(Received 4 June 2008; accepted 10 September 2008; published online 20 October 2008)

The concept of integrable boundary conditions is applied to hydrodynamic type systems. Examples of such boundary conditions for dispersionless Toda systems are obtained. The close relation of integrable boundary conditions with integrable reductions in multifield systems is observed. The problem of consistency of boundary conditions with the Hamiltonian formulation is discussed. Examples of Hamiltonian integrable hydrodynamic type systems on a segment and a semiline are presented. (c) 2008 American Institute of Physics. [DOI: 10.1063/1.2993008]

\section{INTRODUCTION}

The theory of the integrable hydrodynamic type of systems

$$
u_{t}^{i}=v_{j}^{i}(u) u_{x}^{j}, \quad i, j=1,2, \ldots N
$$

was initiated by Dubrovin and Novikov ${ }^{1}$ and Tsarev. ${ }^{2}$ Here in Eq. (1) summation over the repeated indices is assumed and $u$ is an $N$-component column vector of the form $u=\left(u^{1}, u^{2}, \ldots, u^{N}\right)^{t}$. Such systems have a variety of applications in gas dynamics, fluid mechanics, ${ }^{3-6}$ chemical kinetics, Whitham averaging procedure, ${ }^{7-10}$ differential geometry, and topological field theory. We refer to Refs. 11 and 12 for further discussions and references.

In the present article, a problem of finding boundary conditions for hydrodynamic type equations consistent with the integrability property is studied for a special case of the system (1) called dispersionless Toda lattices. ${ }^{13-15}$ Actually we assume that Eq. (1) admits a Lax representation on the algebra

$$
A=\left\{\sum_{-\infty}^{\infty} u_{i}(x) p^{i}: u_{i} \text { decay sufficiently rapidly as } x \rightarrow \pm \infty\right\}
$$

with the following Poisson bracket:

$$
\{f, g\}=p\left(\frac{\partial f}{\partial p} \frac{\partial g}{\partial x}-\frac{\partial f}{\partial x} \frac{\partial g}{\partial p}\right), \quad f, g \in A .
$$

Such equations, for example, appear in the fluid mechanics as reductions in Benney moment equations. ${ }^{3-12}$

Our definition of consistency of boundary conditions with the integrability (see Refs. 16-18) is based on the notion of symmetries. A constraint of the form

\footnotetext{
${ }^{a)}$ Electronic mail: gurses@ fen.bilkent.edu.tr.

${ }^{b}$ Electronic addresses: habibullin_i@mail.rb.ru and habib@fen.bilkent.edu.tr. On leave from Ufa Institute of Mathematics, Russian Academy of Science, Chernyshevskii Str. 112, Ufa 450077, Russia.

c)Electronic mail: zheltukh@metu.edu.tr.
} 


$$
\left.f\left(t, u, u_{[1]}, \ldots, u_{[k]}\right)\right|_{x=x_{0}}=0
$$

where $u_{[j]}=\partial^{j} / \partial x^{j} u$ imposed at some point $x_{0}$ is called a boundary condition at this point. Boundary value problems (1) and (3) [or simply boundary condition (3)] are called consistent with the symmetry

$$
u_{\tau}^{i}=\sigma^{i}\left(t, x, u, u_{[1]}, \ldots, u_{[m]}\right)
$$

if (1) and (4) are compatible under the constraint (3). More precisely, we mean the following: differentiation of (3) with respect to $\tau$ yields

$$
\sum \frac{\partial f}{\partial u_{[n]}^{i}}\left(u_{[n]}^{i}\right)_{\tau}=0
$$

where $\tau$-derivatives are replaced by means of Eq. (4).

Definition 1: Boundary value problems (1) and (3) are consistent with the symmetry (4) if (5) holds identical by means of (3) and its differential consequences obtained by differentiating with respect to $t$.

Note that since constraint (3) is valid only for $x=x_{0}$, it cannot be differentiated with respect to $x$. For this reason, it is convenient to exclude the $x$-derivatives of dependent variable $u$ from our scheme. By solving Eq. (1) for $u_{x}^{i}$ one gets $u_{x}=v^{-1} u_{t}$, where $v^{-1}$ is the matrix inverse to $v_{j}^{i}(u)$. Similarly, $u_{x x}=\left(v^{-1} u_{t}\right)_{x}=\left(v^{-1}\right)_{x} u_{t}+v^{-1}\left(v^{-1}\right)_{t} u_{t}+v^{-2} u_{t t}$ is expressed through $u, u_{t}, u_{t t}$ and so on. As a result one can rewrite boundary condition (3) and symmetry (4) taken at point $x_{0}$ as

$$
f_{1}\left(t, u, u_{t}, \ldots\right)=0
$$

and

$$
u_{\tau}=\sigma_{1}\left(t, x_{0}, u, u_{t}, \ldots\right) .
$$

Now the consistency requirement can be reformulated as follows. Boundary condition (3) is consistent with (4) if differential constraint (6) is consistent with the associated $\tau$-dynamics (7). We call the boundary condition consistent with integrability if it is consistent with an infinite dimensional subspace of symmetries. Hydrodynamic type system given in (1) defines an $N$-dimensional dynamical system and the boundary condition (6) defines a hypersurface in $\mathrm{N}$-dimensional space of functions. Thus, due to the remark above, an integrable boundary condition is closely connected to reductions in the associated system (7) compatible with integrability. ${ }^{15}$ Below we use this important observation in order to find symmetry consistent boundary conditions.

Boundary conditions that passed the symmetry test are then tested for consistency with the conserved quantities, Hamiltonian structures, and the complete integrability property of system (1). ${ }^{19-22}$

It is remarkable that some of the boundary conditions also satisfy these additional requirements and thus allow one to reduce (1) to a completely integrable Hamiltonian system on a segment and a half-line. For more information see Ref. 23 and references therein.

This paper is organized as follows. In Sec. II some integrable boundary conditions for Toda system are derived and it is shown that these boundary conditions are compatible with infinite number of symmetries. The relation between the integrable reductions in $N$-system and the integrable boundary conditions is considered in Sec. III. It is observed that some integrable boundary conditions lead to integrable reductions. In Sec. IV we discuss the compatibility of the integrable boundary conditions found in the previous sections with the Hamiltonian formulation. We show that some boundary conditions are indeed compatible with the Hamiltonian formulation and also with an infinite class of symmetries. In all sections up to Sec. IV only $N=2$ systems are considered. In Sec. V we study $N=3$ systems which give other examples of hydrodynamic type equa- 
tions. For this case, some integrable boundary conditions compatible with infinite number of symmetries and boundary conditions compatible with the Hamiltonian formulation are found.

\section{INTEGRABLE BOUNDARY CONDITIONS FOR THE TODA SYSTEM}

In this section we study the well known example of integrable model ${ }^{24}$

$$
\begin{gathered}
S_{t}=P_{x}, \\
P_{t}=P S_{x}
\end{gathered}
$$

called Toda system, admitting the Lax representation on the algebra of Lourent series (2)

$$
L_{t}=\left\{(L)_{\geq 0}, L\right\},
$$

where

$$
L=p+S+P p^{-1} .
$$

The corresponding hierarchy of symmetries of the Toda system (8) is

$$
L_{t_{n}}=\left\{L,\left(L^{n}\right)_{\geq 0}\right\} .
$$

Recursion operator corresponding to the above hierarchy is (for calculation of recursion operator see Refs. 15 and 25)

$$
\mathfrak{R}=\left(\begin{array}{ll}
S & 2+P_{x} D_{x}^{-1} \cdot P^{-1} \\
2 P & S+S_{x} P D_{x}^{-1} \cdot P^{-1}
\end{array}\right) .
$$

In some cases, it is convenient to consider the Toda system (8) in other variables. We write the Lax function (10) as $L=p^{-1}(p+u)(p+v)$, that is,

$$
S=u+v, \quad P=u v .
$$

Then the Toda system (9) gives

$$
\begin{aligned}
& u_{t}=u v_{x}, \\
& v_{t}=v u_{x} .
\end{aligned}
$$

Let us find boundary conditions compatible with an infinite number of symmetries from the hierarchy (11). As a boundary, we take $x=0$. First we find boundary conditions compatible with the first symmetry of the hierarchy (11). Assume that the boundary condition depends on $P$ and $S$ and can be solved with respect to $S$. So the boundary condition can be written as

$$
S=F(P), \quad x=0 .
$$

Lemma 1: On the boundary $x=0$, the boundary condition of the form (15) compatible with the first symmetry of the hierarchy (11)

$$
\begin{gathered}
S_{t_{1}}=2 S P_{x}+2 P S_{x}, \\
P_{t_{1}}=2 P P_{x}+2 S P S_{x}
\end{gathered}
$$

is given by

$$
P=\frac{(S+c)^{2}}{4}, \quad x=0 .
$$


Proof: The boundary condition (15) is compatible with the symmetry (16) if on the boundary $x=0$

$$
S_{t_{1}}=F^{\prime}(P) P_{t_{1}}
$$

for all solutions of Eq. (8). Let us find functions $F$ for which the above equality holds. We rewrite the symmetry (16) in terms of variables $S, P$, and their $t$ derivatives using Eq. (8)

$$
\begin{gathered}
S_{t_{1}}=2 S S_{t}+2 P_{t}, \\
P_{t_{1}}=2 P S_{t}+2 S P_{t} .
\end{gathered}
$$

Then we substitute $S_{t_{1}}$ and $P_{t_{1}}$ into (18), so

$$
2 S S_{t}+2 P_{t}=F^{\prime}(P)\left(2 P S_{t}+2 S P_{t}\right) .
$$

From (15) it follows that $S_{t}=F^{\prime}(P) P_{t}$, so

$$
2 S F^{\prime}(P) P_{t}+2 P_{t}=F^{\prime}(P)\left(2 P F^{\prime}(P) P_{t}+2 S P_{t}\right) .
$$

Hence,

$$
F^{\prime 2}(P)=\frac{1}{P}
$$

The above equation has a solution (17).

It is convenient to write the boundary condition (17) as

$$
P=\frac{S^{2}}{4}, \quad x=0 .
$$

By shifting $S$, the Toda system (8) is invariant with respect to such shift.

Lemma 2: All the symmetries of the hierarchy (11) are compatible with the boundary condition (23).

Proof: The boundary condition (23) is compatible with an evolution symmetry

$$
\left(\begin{array}{l}
S \\
P
\end{array}\right)_{\tau}=\left(\begin{array}{l}
\sigma \\
\pi
\end{array}\right)
$$

if $\pi=\frac{1}{2} S \sigma$ for $P=\frac{1}{4} S^{2}$. That is, under the constraint (23) the symmetry (24) should take the form

$$
\left(\begin{array}{l}
S_{\tau} \\
\frac{1}{2} S S_{\tau}
\end{array}\right)=\left(\begin{array}{l}
\sigma \\
\frac{1}{2} S \sigma
\end{array}\right)
$$

Evidently, the first symmetry of the hierarchy (11) has such a form. Let us show that the recursion operator (12) preserves the property (25). On the boundary $x=0$, we rewrite the recursion operator (12) in terms of $t$ derivatives using the Toda system (8) as follows:

$$
\mathfrak{R}=\left(\begin{array}{ll}
S+S_{t} D_{t}^{-1} & 2 \\
2 P+P_{t} D_{t}^{-1} & S
\end{array}\right) .
$$

Applying the recursion operator (26) into a symmetry (25), we obtained a symmetry

$$
\left(\begin{array}{l}
S \\
P
\end{array}\right)_{\tilde{\tau}}=\left(\begin{array}{l}
\widetilde{\sigma} \\
\frac{1}{2} S \widetilde{\sigma}
\end{array}\right)
$$


We also have the following boundary condition compatible with the hierarchy (11).

Lemma 3: On the boundary $x=0$, the boundary condition

$$
P=0
$$

is compatible with all symmetries of the hierarchy (11).

The above lemma is proved in the same way as Lemma 2.

Another boundary condition comes from consideration of odd and even solutions of the Toda system (8). This boundary condition is not compatible with all symmetries of the hierarchy (11) but only with even ones.

Lemma 4: On the boundary $x=0$, the boundary condition

$$
S=0
$$

is compatible with all even numbered symmetries of the hierarchy (11).

The above lemma is proved in the same way as Lemma 2 using the square of the recursion operator (26).

\section{INTEGRABLE REDUCTIONS}

Let us consider other equations admitting a Lax representation on the algebra (2). For a Lax function $L=p^{-1}\left(p-u_{N}\right)\left(p-u_{N-1}\right) \cdots\left(p-u_{1}\right)$, where $N>2$, we consider the Lax equation,

$$
L_{t}=\left\{L,(L)_{\geq 0}\right\}
$$

and an infinite hierarchy of symmetries

$$
L_{t_{n}}=\left\{L,\left(L^{n}\right)_{\geq 0}\right\}, \quad n=1,2, \ldots .
$$

For such equations we cannot directly find boundary conditions compatible with symmetries (see Sec. V). So we use integrable reductions. ${ }^{15}$

Definition 2: A reduction in an integrable equation is called integrable if reduced equation is also integrable. That is, the reduced equation admits an infinite hierarchy of symmetries.

In Ref. 15 it was shown that the following reductions

$$
\begin{gathered}
u_{N}=u_{N-1}=\cdots=u_{i}=0, \quad i \geq 2, \\
u_{N}=u_{N-1}=\cdots=u_{j}, \quad j \geq 1
\end{gathered}
$$

of the above equations are integrable. We note that for these reductions the symmetries of the reduced equation are obtained by the reduction in the symmetries of the original system.

If we have an integrable reduction such that symmetries of the reduced system are obtained by the reduction in the symmetries of the original system, then the reduction can be taken as integrable boundary conditions. Indeed, the original system is invariant under the hierarchy of symmetries and the reduced system is invariant under the symmetries. Since reduction can be recovered from the original system and the reduced system, it is also invariant under the symmetries. So, taking the reductions (32) as boundary conditions we obtain symmetry invariant boundary conditions.

Theorem 1: For a system (30) the boundary condition $\left.\left(u_{N}=u_{N-1}=\cdots=u_{i}\right)\right|_{x=a}=0$ or $\left(u_{N}\right.$ $\left.=u_{N-1}=\cdots=u_{j}\right)\left.\right|_{x=a}$ (taking $x=a$ as the boundary) is integrable.

Let us take boundary conditions obtained in Sec. II. The condition $\left.\left(P=S^{2} / 4\right)\right|_{x=0}$ in $u, v$ variables (13) is $\left.(u-v)\right|_{x=0}=0$. It corresponds to a reduction $u=v$. The condition $\left.P\right|_{x=0}=0$ in $u, v$ variables is $\left.(u v)\right|_{x=0}=0$. It corresponds to a reduction $u=0$ (or $v=0$ ). The condition $\left.S\right|_{x=0}=0$ in $u, v$ variables is $\left.(u+v)\right|_{x=0}=0$. It does not correspond to reductions considered above.

Remark: If we take a reduction mentioned above as a boundary condition, then we can consider the corresponding reduced system. Solutions of the reduced system obviously satisfy the 
main system equations and the boundary condition. For Toda system, the reduction $P=0$ leads to the equation

$$
S_{t}=0 .
$$

Its solution $S=f(x)$, for any differentiable function $f$, gives the solution of Toda system (8) satisfying the corresponding boundary condition (28). The reduction $P=S^{2} / 4$ leads to the Hopf equation,

$$
S_{t}=\frac{1}{2} S S_{x} .
$$

Its solution $S=h(2 x+t S)$ gives the solution of Toda system (8) satisfying the corresponding boundary condition. Here $h$ is any differentiable function of $x$ and $t$. To find a solution of $N$-system satisfying the integrable boundary condition, the method described above is very effective. We take the corresponding reduction and the corresponding reduced $(N-1)$ system. Solving the reduced system gives automatically the solution of the $N$-system, satisfying the integrable boundary condition.

\section{HAMILTONIAN REPRESENTATION OF THE INTEGRABLE BOUNDARY VALUE PROBLEMS}

To obtain the Hamiltonian formulation of the Toda system (8), we use its Lax representation on the algebra (2).

We define, for the algebra of Lourent series (2), a trace functional

$$
\operatorname{tr} f=\int_{-\infty}^{\infty} u_{0} d x, \quad f \in A, \quad f=\sum_{-\infty}^{\infty} u_{i}(x) p^{i}
$$

and a nondegenerate ad-invariant pairing

$$
(f, g)=\operatorname{tr}(f \cdot g), \quad f, g \in A .
$$

Thus we have a Poisson algebra with a commutative multiplication and unity, the multiplication satisfies the derivation property with respect to the Poisson bracket, and the algebra is equipped with a nondegenerate ad-invariant pairing. Following Ref. 14 we can define an infinite family of Poisson structures for smooth functions on the algebra $A$. A function $F$ on $A$ is smooth if there is a map $d F: A \rightarrow A$ such that

$$
\left.F^{\prime}\right|_{t=0}(f+t g)=(d F(f), g), \quad f, g \in A .
$$

The following theorem (Ref. 14 see also Refs. 26 and 27) holds.

Theorem 2: Let A be a Poisson algebra with unity, commutative multiplication, Poisson bracket $\{. .$,$\} , and nondegenerate, ad-invariant pairing (...). Assume that the multiplication satisfies$ the derivation property with respect to the Poisson bracket and is symmetric with respect to the pairing $(f g, h)=(f, g h)$. If $R: A \rightarrow A$ is a classical r-matrix, then for smooth functions $F$ and $G$ on A:

(a)

$$
\{F, G\}_{(n)}(f)=\left(f,\left\{R\left(f^{n+1} d F(f)\right), d G(f)\right\}\right)+\left(f,\left\{d F(f), R\left(f^{n+1} d G(f)\right)\right\}\right)
$$

defines a Poisson structure for each integer $n \geq-1$.

(b) The structures $\{.,\}_{(n)}$ are compatible with each other (their sum is again a Poisson structure).

A linear operator $R: A \rightarrow A$ is a classical $r$-matrix if the bracket 


$$
[f, g]=\frac{1}{2}(\{R f, g\}+\{f, R g\})
$$

is a Lie bracket.

To apply the above theorem, we take an $r$-matrix

$$
R=\frac{1}{2}\left(P_{\geq 0}-P_{\leq-1}\right),
$$

where $P_{\geq 0}$ and $P_{\leq-1}$ are projectors on Poisson subalgebras

$$
A_{\geq 0}=\left\{u=\sum_{0}^{\infty} u_{i} p^{i}: u \in A\right\} \quad \text { and } \quad A_{\leq 0}=\left\{u=\sum_{-\infty}^{-1} u_{i} p^{i}: u \in A\right\},
$$

respectively. Note that the Lax equation (9) is

$$
L_{t}=\{R(L), L\},
$$

where $L=p+S+P p^{-1}$.

Using the Poisson structures given by Theorem 2 we obtain bi-Hamiltonian formulation of the Toda lattice.

The submanifold $M=\left\{L \in A: L=p+S+P p^{-1}\right\}$ is a Poisson submanifold for the Poisson structure (37) with $n=-1$. Restricting this structure on $M$ we obtain the following Hamiltonian operator:

$$
\mathfrak{D}_{-1}=\left(\begin{array}{ll}
0 & P D_{x}+P_{x} \\
P D_{x} & 0
\end{array}\right) .
$$

We have first Hamiltonian formulation for (8)

$$
\left(\begin{array}{l}
S \\
P
\end{array}\right)_{t}=\mathfrak{D}_{-1}\left(\begin{array}{l}
\delta \mathcal{H}_{-1} / \delta S \\
\delta \mathcal{H}_{-1} / \delta P
\end{array}\right)
$$

where

$$
\mathcal{H}_{-1}=\frac{1}{2} \operatorname{tr} L^{2}, \quad \text { that is, } \quad \mathcal{H}_{-1}=\frac{1}{2} \int_{-\infty}^{\infty}\left(S^{2}+2 P\right) d x
$$

The second Hamiltonian operator can be obtained by restricting the Poisson structure (37) with $n=0$ on the submanifold $M$ or by application of the recursion operator (12) to the Hamiltonian operator (40). The second Hamiltonian operator is

$$
\mathfrak{D}_{0}=\left(\begin{array}{ll}
2 P D_{x}+P_{x} & S P D_{x}+S P_{x} \\
S P D_{x}+S_{x} P & P^{2} D_{x}+P P_{x}
\end{array}\right) .
$$

The corresponding Hamiltonian functional is

$$
\mathcal{H}_{0}=\operatorname{tr} L, \quad \text { that is, } \quad \mathcal{H}_{0}=\int_{-\infty}^{\infty} S d x .
$$

Since Hamiltonian operators $\mathfrak{D}_{-1}$ and $\mathfrak{D}_{0}$ are compatible, we have a bi-Hamiltonian representation of Eq. (8).

In $u, v$ variables (13) the Hamiltonian operators and functionals take form

$$
\mathfrak{B}_{-1}=\frac{u v}{(u-v)^{2}}\left(\begin{array}{cc}
-2 u & u+v \\
u+v & -2 v
\end{array}\right) D_{x}
$$




$$
+\frac{1}{(u-v)^{3}}\left(\begin{array}{ll}
2 u v^{2} u_{x}-u^{3} v_{x}-u^{2} v v_{x} & u^{2} v v_{x}+u^{3} v_{x}-2 u v^{2} u_{x} \\
2 u v^{2} v_{x}-u v^{2} u_{x}-v^{3} u_{x} & v^{3} u_{x}+u v^{2} u_{x}-2 u^{2} v v_{x}
\end{array}\right)
$$

and

$$
\begin{gathered}
\mathcal{G}_{-1}=\int_{-\infty}^{\infty}\left(u^{2}+v^{2}+4 u v\right) d x, \\
\mathfrak{B}_{0}=\left(\begin{array}{cc}
0 & u v_{x}+u v D_{x} \\
v u_{x}+u v D_{x} & 0
\end{array}\right),
\end{gathered}
$$

and

$$
\mathcal{G}_{0}=\int_{-\infty}^{\infty}(u+v) d x .
$$

A different approach was used in Ref. 13 to obtain the Hamiltonian operator $\mathfrak{B}_{0}$ (see also references in Ref. 13). The explicit expressions of an infinite number of conservation laws for the Toda system (14) was given in Ref. 13,

$$
Q_{n, t}=F_{n, x}, \quad n=1,2 \ldots,
$$

where

$$
Q_{n}=\sum_{j=0}^{n}\left(\begin{array}{l}
n \\
j
\end{array}\right)^{2} u^{j} v^{n-j}, \quad n=1,2,3 \ldots
$$

and

$$
F_{n}=\sum_{j=0}^{n} \frac{n-j}{j+1}\left(\begin{array}{c}
n \\
j
\end{array}\right)^{2} u^{j+1} v^{n-j}, \quad n=1,2,3 \ldots .
$$

The conserved quantities $\mathcal{Q}_{n}=\int_{-\infty}^{\infty} Q_{n} d x$ are in involution with respect to the Hamiltonian operators $\mathfrak{B}_{-1}$ and $\mathfrak{B}_{0}$. One can easily check if the boundary conditions preserve the conserved quantities.

Lemma 5: For the Toda system (14) with the boundary condition

(a) $\left.(u-v)\right|_{x=0}=0\left(\left.\left(P=S^{2} / 4\right)\right|_{x=0}\right)$ the above conservation laws are not preserved;

(b) $\left.u v\right|_{x=0}=0\left(\left.P\right|_{x=0}=0\right)$ the quantities

$$
\int_{0}^{\infty} \mathcal{Q}_{n} d x, \quad n=1,2,3 \ldots
$$

are conserved; and

(c) $\left.(u+v)\right|_{x=0}=0\left(\left.S\right|_{x=0}=0\right)$ the quantities

$$
\int_{0}^{\infty} \mathcal{Q}_{n} d x, \quad n=2,4,6 \ldots
$$

are conserved.

We can use the above Hamiltonian operators to obtain the Hamiltonian representation of some of the boundary value problems.

Theorem 3: The Toda system (14) on a segment [0,1] with boundary conditions 


$$
\left.u v\right|_{x=0}=0 \text { and }\left.\quad u v\right|_{x=1}=0
$$

admits the bi-Hamiltonian representation with Hamiltonian operators $\mathfrak{B}_{(n)}, n=-1,0$, and Hamiltonians

$$
\overline{\mathcal{G}}_{-1}=\int_{0}^{1}\left(u^{2}+v^{2}+4 u v\right) d x=\int_{-\infty}^{\infty}\left(u^{2}+v^{2}+4 u v\right) \theta(x) \theta(1-x) d x
$$

and

$$
\overline{\mathcal{G}}_{0}=\int_{0}^{1}(u+v) d x=\int_{-\infty}^{\infty}(u+v) \theta(x) \theta(1-x) d x,
$$

respectively, where $\theta(x)$ is the Heaviside step function.

Proof: The Hamiltonian equations

$$
\left(\begin{array}{l}
u \\
v
\end{array}\right)_{t}=\mathfrak{B}_{n}\left(\begin{array}{l}
\delta \overline{\mathcal{G}}_{n} / \delta u \\
\delta \overline{\mathcal{G}}_{n} / \delta v
\end{array}\right), \quad n=-1,0
$$

are for $n=-1$

$$
\begin{aligned}
& u_{t}=u v_{x}-\frac{u v}{u-v}(\delta(x)-\delta(1-x)), \\
& v_{t}=v u_{x}+\frac{u v}{u-v}(\delta(x)-\delta(1-x))
\end{aligned}
$$

and for $n=0$

$$
\begin{aligned}
& u_{t}=u v_{x}+u v(\delta(x)-\delta(1-x)), \\
& v_{t}=v u_{x}+u v(\delta(x)-\delta(1-x)),
\end{aligned}
$$

where $x \in[0,1]$. Under the boundary conditions $\left.u v\right|_{x=0}=0$ and $\left.u v\right|_{x=1}=0$ we have the Toda system (14) on $[0,1]$. Note that the Poisson brackets are given by

$$
\{\mathcal{K}, \mathcal{N}\}=\int_{-\infty}^{\infty}\left(\begin{array}{c}
\delta \mathcal{K} / \delta u \\
\delta \mathcal{K} / \delta v
\end{array}\right) \mathfrak{B}_{(n)}\left(\begin{array}{l}
\delta \mathcal{N} / \delta u \\
\delta \mathcal{N} / \delta v
\end{array}\right)
$$

where $n=-1,0$.

\section{INTEGRABLE BOUNDARY CONDITIONS FOR THE THREE FIELD SYSTEMS}

Let us consider a three field hydrodynamic type system on the algebra (2). We take a Lax function

$$
L=p^{2}+S p+P+Q p^{-1}
$$

We can construct two integrable hierarchies with this Lax function.

The first hierarchy is given by

$$
L_{t}=\left\{\left(L^{n+1 / 2}\right)_{\geq 0}, L\right\}, \quad n=0,1,2, \ldots,
$$

the first equation of the hierarchy is

$$
S_{t}=P_{x}-\frac{1}{2} S S_{x},
$$




$$
\begin{gathered}
P_{t}=Q_{x}, \\
Q_{t}=\frac{1}{2} Q S_{x} .
\end{gathered}
$$

The second hierarchy is given by

$$
L_{t}=\left\{\left(L^{n}\right)_{\geq 0}, L\right\}, \quad n=1,2,3 \ldots,
$$

the first equation of the hierarchy is

$$
\begin{gathered}
S_{t}=2 Q_{x}, \\
P_{t}=S Q_{x}+Q S_{x}, \\
Q_{t}=Q P_{x} .
\end{gathered}
$$

We also have a recursion operator ${ }^{15}$ of the hierarchies (63) and (65),

$$
\left(\begin{array}{lll}
P-\frac{1}{4} S^{2}+\left(\frac{1}{2} P_{x}-\frac{1}{4} S S_{x}\right) D_{x}^{-1} & \frac{1}{2} S & 3+2 Q_{x} D_{x}^{-1} Q^{-1} \\
\frac{3}{2} Q+\frac{1}{2} Q_{x} D_{x}^{-1} & P & 2 S+(S Q)_{x} D_{x}^{-1} Q^{-1} \\
\frac{1}{4} S Q+\frac{1}{4} S_{x} Q D_{x}^{-1} & \frac{3}{2} Q & P+Q P_{x} D_{x}^{-1} Q^{-1}
\end{array}\right)
$$

The bi-Hamiltonian representation of Eqs. (64) and (66) is obtained by restricting the Poisson structure (37) with $n=-1$ and $n=0$ on the submanifold $M=\left\{L \in A: L=p^{2}+S p+P+Q p^{-1}\right\}$. So we have Hamiltonian operators

$$
\mathfrak{C}_{-1}=\left(\begin{array}{lll}
2 D_{x} & 0 & 0 \\
0 & 0 & Q D_{x}+Q_{x} \\
0 & Q D_{x} & 0
\end{array}\right)
$$

and

$$
\mathfrak{C}_{0}=\left(\begin{array}{lll}
\left(2 P-\frac{1}{2} S^{2}\right) D_{x}+P_{x}-\frac{1}{2} S S_{x} & 3 Q D_{x}+2 Q_{x} & \frac{1}{2} Q D_{x}+\frac{1}{2} S Q_{x} \\
Q D_{x}+Q_{x} & 2 S Q D_{x}+S Q_{x}+Q S_{x} & P Q D_{x}+P D_{x} \\
\frac{1}{2} S Q D_{x}+\frac{1}{2} Q S_{x} & P D_{x}+P_{x} Q & \frac{3}{2} Q^{2} D_{x}+\frac{3}{2} Q Q_{x}
\end{array}\right) .
$$

Equation (64) can be written as

$$
\left(\begin{array}{l}
S \\
P \\
Q
\end{array}\right)_{t}=\mathfrak{C}_{-1}\left(\begin{array}{c}
\delta \overline{\mathcal{H}}_{-1} / \delta S \\
\delta \overline{\mathcal{H}}_{-1} / \delta P \\
\delta \overline{\mathcal{H}}_{-1} / \delta Q
\end{array}\right)=\mathfrak{C}_{0}\left(\begin{array}{c}
\delta \overline{\mathcal{H}}_{0} / \delta S \\
\delta \overline{\mathcal{H}}_{0} / \delta P \\
\delta \overline{\mathcal{H}}_{0} / \delta Q
\end{array}\right)
$$

where

$$
\overline{\mathcal{H}}_{-1}=\frac{2}{3} \operatorname{tr} L^{3 / 2}, \quad \text { that is, } \quad \overline{\mathcal{H}}_{-1}=\int_{-\infty}^{\infty}\left(Q+\frac{1}{2} S P-\frac{1}{24} S^{3}\right) d x
$$

and

$$
\overline{\mathcal{H}}_{0}=2 \operatorname{tr} L^{1 / 2}, \quad \text { that is, } \quad \overline{\mathcal{H}}_{0}=\int_{-\infty}^{\infty} S d x
$$

Equation (66) can be written as 


$$
\left(\begin{array}{l}
S \\
P \\
Q
\end{array}\right)_{t}=\mathfrak{C}_{-1}\left(\begin{array}{c}
\delta \tilde{\mathcal{H}}_{-1} / \delta S \\
\delta \tilde{\mathcal{H}}_{-1} / \delta P \\
\delta \tilde{\mathcal{H}}_{-1} / \delta Q
\end{array}\right)=\mathfrak{C}_{0}\left(\begin{array}{c}
\delta \tilde{\mathcal{H}}_{0} / \delta S \\
\delta \tilde{\mathcal{H}}_{0} / \delta P \\
\delta \tilde{\mathcal{H}}_{0} / \delta Q
\end{array}\right),
$$

where

$$
\widetilde{\mathcal{H}}_{-1}=\frac{1}{2} \operatorname{tr} L^{2}, \quad \text { that is, } \quad \widetilde{\mathcal{H}}_{-1}=\int_{-\infty}^{\infty}\left(S Q+\frac{1}{2} P^{2}\right) d x
$$

and

$$
\widetilde{\mathcal{H}}_{0}=\operatorname{tr} L, \quad \text { that is, } \quad \tilde{\mathcal{H}}_{0}=\int_{-\infty}^{\infty} P d x
$$

We can give both hierarchies in modified variables, writing the Lax function (62) as $L$ $=p^{-1}(p-u)(p-v)(p-w)$, that is,

$$
\begin{gathered}
S=u+v+w, \\
P=u v+u w+v w, \\
Q=u v w .
\end{gathered}
$$

It is quite difficult to find integrable boundary condition directly for three field systems. For example, consider hierarchy (63). In the following lemmas, we use $P, Q, R$ variables since symmetries and recursion operator have a simple form in these variables.

Lemma 6: Let $x=0$ be the boundary. The boundary conditions of the form $P=F(S)$ and $Q$ $=G(S)$ are compatible with the first symmetry of the hierarchy(63) if the functions $F$ and $G$ satisfy the following differential equations:

$$
\begin{aligned}
& \frac{3}{2} S\left(F^{\prime}\right)^{2}+3 F^{\prime} G^{\prime}-\frac{3}{4} F^{\prime} S^{2}-3 G^{\prime} S-\frac{3}{2} G=0, \\
& \frac{3}{2} S F^{\prime} G^{\prime}+3\left(G^{\prime}\right)^{2}-\frac{3}{2} F^{\prime} G-\frac{3}{4} G^{\prime} S^{2}-\frac{3}{4} S G=0 .
\end{aligned}
$$

Proof: The first symmetry of the hierarchy (63) is

$$
\begin{gathered}
S_{t_{1}}=\frac{3}{2}\left(P-\frac{1}{4} S^{2}\right)\left(P_{x}-\frac{1}{2} S S_{x}\right)+\frac{3}{2} S Q_{x}+\frac{3}{2} S_{x} Q, \\
P_{t_{1}}=\frac{3}{2} P Q_{x}+\frac{3}{2} P_{x} Q^{\frac{3}{4}} Q S S_{x}+\frac{3}{8} S^{2} Q_{x}, \\
Q_{t_{1}}=\frac{1}{4} S Q\left(P_{x}-\frac{1}{2} S S_{x}\right)+\frac{1}{4} Q\left(P-\frac{1}{4} S^{2}\right)+\frac{3}{2} Q Q_{x}+\frac{1}{2} Q P S_{x}+\frac{1}{2} Q S P_{x} .
\end{gathered}
$$

Differentiating the boundary conditions $P=F(S)$ and $Q=G(S)$ with respect to the above symmetry and expressing all the $x$ derivatives in terms of $t$ derivatives using Eq. (64), we obtain Eqs. (77) and (78).

Lemma 7: Let $x=0$ be the boundary. The boundary condition of the form $S=F(P, Q)$ is compatible with the first symmetry of the hierarchy (63) if function $F$ satisfies the following differential equations:

$$
\frac{3}{2}\left(P-\frac{1}{4} F^{2}\right) F_{P}+\frac{3}{2} F=\frac{3}{2} P F_{P}+\frac{3}{2} Q F_{P}^{2}+\frac{3}{8} Q F_{P}^{2}+\frac{3}{8} F^{2} F_{P}+\frac{1}{4} Q F F_{Q}+\frac{3}{2} Q F_{Q}+\frac{1}{2} F F_{P} F_{Q},
$$




$$
\frac{3}{2}\left(P-\frac{1}{4} F^{2}\right) F_{Q}+\frac{3}{2}=\frac{3}{2} Q F_{P} F_{Q}+\frac{3}{2} F F_{P}+\frac{1}{2}\left(P-\frac{1}{4} S^{2}\right) F_{Q}+P F_{Q}+\frac{1}{2} Q F F_{Q}^{2}+\frac{1}{2} F^{2} F_{Q} .
$$

Proof: We differentiate the boundary condition $S=F(P, Q)$ with respect to the symmetry (63) and express all the $x$ derivatives in terms of $t$ derivatives using Eq. (64). Then separating terms containing $P_{t}$ and $Q_{t}$, we obtain Eqs. (80) and (81).

The differential equations obtained in the above lemmas are nonlinear partial differential equations which are rather complicated. So, to obtain integrable boundary conditions it is easy to use integrable reductions discussed in Sec. III. Let $x=0$ be a boundary.

(a) Integrable reduction $u=v$ gives integrable boundary condition $\left.u\right|_{x=0}=\left.v\right|_{x=0}$ or $\left(S^{3} Q-S^{2} P^{2}\right.$ $\left.+4 Q^{3}-18 S P Q+27 Q^{2}\right)\left.\right|_{x=0}=0$ (condition on coefficients of cubic equation to have two equal roots) in $S, P, Q$ variables.

(b) Integrable reduction $u=v=w$ gives integrable boundary conditions $\left.u\right|_{x=0}=\left.v\right|_{x=0}=\left.w\right|_{x=0}$ or $\left.P\right|_{x=0}=\left.\frac{1}{3} S^{2}\right|_{x=0},\left.Q\right|_{x=0}=\left.\frac{1}{27} S^{3}\right|_{x=0}$ (condition on coefficients of cubic equation to have all roots equal).

(c) Integrable reduction $u=0$ gives integrable boundary condition $\left.u\right|_{x=0}=0$ or $\left.Q\right|_{x=0}=0$.

(d) Integrable reduction $u=0, v=0$ gives integrable boundary conditions $\left.u\right|_{x=0}=0,\left.v\right|_{x=0}=0$ or $\left.P\right|_{x=0}=0,\left.Q\right|_{x=0}=0$.

To obtain boundary value problems that admit bi-Hamiltonian representation we modify Hamiltonian functions, as in the case of Toda system. We use $S, P, Q$ variables, the Hamiltonian operators have simpler form in this variables.

For Eq. (64) we have the following.

Theorem 4: Equation (64) on a segment $[0,1]$ with boundary conditions

$$
\left.\left(P-\frac{1}{4} S^{2}\right)\right|_{x=0}=0,\left.Q\right|_{x=0}=0 \quad \text { and }\left.\left(P-\frac{1}{4} S^{2}\right)\right|_{x=1}=0,\left.Q\right|_{x=1}=0
$$

admits the bi-Hamiltonian representation with Hamiltonian operators (68) and (69), and Hamiltonians

$$
\overline{\overline{\mathcal{H}}}_{-1}=\int_{-\infty}^{\infty}\left(Q+\frac{1}{2} S P-\frac{1}{24} S^{3}\right) \theta(x) \theta(1-x) d x
$$

and

$$
\overline{\overline{\mathcal{H}}}_{0}=\int_{-\infty}^{\infty} S \theta(x) \theta(1-x) d x,
$$

respectively, where $\theta(x)$ is the Heaviside step function.

Proof: The Hamiltonian equations

$$
\left(\begin{array}{l}
S \\
P \\
Q
\end{array}\right)_{t}=\mathfrak{C}_{n}\left(\begin{array}{c}
\delta \overline{\overline{\mathcal{H}}}_{n} / \delta S \\
\delta \overline{\overline{\mathcal{H}}}_{n} / \delta P \\
\delta \overline{\overline{\mathcal{H}}}_{n} / \delta Q
\end{array}\right), \quad n=-1,0
$$

are for $n=-1$ 


$$
\begin{gathered}
S_{t}=P_{x}-\frac{1}{2} S S_{x}+\left(P-\frac{1}{4} S^{2}\right)(\delta(x)-\delta(1-x)), \\
P_{t}=Q_{x}+\frac{1}{2} S Q(\delta(x)-\delta(1-x)), \\
Q_{t}=\frac{1}{2} Q S_{x}+Q(\delta(x)-\delta(1-x))
\end{gathered}
$$

and for $n=0$

$$
\begin{gathered}
S_{t}=P_{x}-\frac{1}{2} S S_{x}+\left(2 P-\frac{1}{2} S^{2}\right)(\delta(x)-\delta(1-x)), \\
P_{t}=Q_{x}+Q(\delta(x)-\delta(1-x)), \\
Q_{t}=\frac{1}{2} Q S_{x}+\frac{1}{2} S Q(\delta(x)-\delta(1-x)),
\end{gathered}
$$

where $x \in[0,1]$. Under the boundary conditions (82) we have Eq. (64) on [0,1].

The boundary conditions (82) are symmetry integrable.

Lemma 8: All the symmetries of the hierarchy (63) are compatible with the boundary condition (82).

Proof: The boundary condition (82) is compatible with an evolution symmetry

$$
\left(\begin{array}{l}
S \\
P \\
Q
\end{array}\right)_{\tau}=\left(\begin{array}{l}
\sigma \\
\pi \\
\kappa
\end{array}\right)
$$

if $\pi=\frac{1}{2} S \sigma$ and $\kappa=0$ for $P=\frac{1}{4} S^{2}$ and $Q=0$ on the boundary $x=0$. That is, under the conditions (82) the symmetry $(88)$ should take the form

$$
\left(\begin{array}{c}
S_{\tau} \\
\frac{1}{2} S S_{\tau} \\
0
\end{array}\right)=\left(\begin{array}{c}
\sigma \\
\frac{1}{2} S \sigma \\
0
\end{array}\right)
$$

One can check that the first symmetry of the hierarchy (63) has such a form. Let us show that the recursion operator (67) preserves the form (89). On the boundary $x=0$, we rewrite the recursion operator (67) in terms of $t$ derivatives using Eq. (64) as follows:

$$
\left(\begin{array}{lll}
P-\frac{1}{4} S^{2}-\frac{1}{4} S_{t} D_{t}^{-1} S & \frac{1}{2} S+\frac{1}{2} S_{t} D_{t}^{-1} & 3+P_{t} D_{t}^{-1} \\
\frac{3}{2} Q-\frac{1}{4} P_{t} D_{t}^{-1} S & P+\frac{1}{2} P_{t} D_{t}^{-1} & 2 S+\left(\frac{1}{2} S P_{t}+Q_{t}\right) D_{t}^{-1} \\
\frac{1}{4} S Q-\frac{1}{4} Q_{t} D_{t}^{-1} S & \frac{3}{2} Q+\frac{1}{2} Q_{t} D_{t}^{-1} & P+\frac{1}{2} Q P_{t} D_{t}^{-1}
\end{array}\right)
$$

Applying the recursion operator (90) to a symmetry (89) we obtained a symmetry

$$
\left(\begin{array}{l}
S_{\tilde{\tau}} \\
\frac{1}{2} S S_{\tilde{\tau}} \\
0
\end{array}\right)=\left(\begin{array}{l}
\tilde{\sigma} \\
\frac{1}{2} S \widetilde{\sigma} \\
0
\end{array}\right)
$$

For Eq. (66) we have the following.

Theorem 5: Equation (66) on a segment [0,1] with boundary conditions

$$
\left.Q\right|_{x=0}=0 \quad \text { and }\left.\quad Q\right|_{x=1}=0
$$


admits the bi-Hamiltonian representation with Hamiltonian operators (68) and (69), and Hamiltonians

$$
\widetilde{\tilde{\mathcal{H}}}_{-1}=\int_{-\infty}^{\infty}\left(S Q+\frac{1}{2} P^{2}\right) \theta(x) \theta(1-x) d x
$$

and

$$
\tilde{\widetilde{\mathcal{H}}}_{0}=\int_{-\infty}^{\infty} P \theta(x) \theta(1-x) d x,
$$

respectively, where $\theta(x)$ is the Heaviside step function.

Proof: The Hamiltonian equations

$$
\left(\begin{array}{l}
S \\
P \\
Q
\end{array}\right)_{t}=\mathfrak{C}_{n}\left(\begin{array}{c} 
\\
\delta \tilde{\widetilde{H}}_{n} / \delta S \\
\delta \tilde{\widetilde{\mathcal{H}}}_{n} / \delta P \\
\delta \tilde{\widetilde{\mathcal{H}}}_{n} / \delta Q
\end{array}\right), \quad n=-1,0
$$

are for $n=-1$

$$
\begin{gathered}
S_{t}=2 Q_{x}+2 Q(\delta(x)-\delta(1-x)), \\
P_{t}=S Q_{x}+Q S_{x}+P Q(\delta(x)-\delta(1-x)), \\
Q_{t}=Q P_{x}+S Q(\delta(x)-\delta(1-x))
\end{gathered}
$$

and for $n=0$

$$
\begin{gathered}
S_{t}=2 Q_{x}+Q(\delta(x)-\delta(1-x)), \\
P_{t}=S Q_{x}+Q S_{x}+2 S Q(\delta(x)-\delta(1-x), \\
Q_{t}=Q P_{x}+P Q(\delta(x)-\delta(1-x)),
\end{gathered}
$$

where $x \in[0,1]$. Under the boundary conditions (92) we have Eq. (66) on $[0,1]$.

In the same way as in Lemma 8, one can show that the boundary condition (92) is symmetry integrable. This case is similar to the case of Toda system (the boundary condition $\left.Q\right|_{\Gamma}=0$ in modified variables is $\left.\left.u v w\right|_{\Gamma}=0\right)$.

\section{CONCLUSION}

In this article we studied the problem of integrable boundary conditions for hydrodynamic type integrable systems. To our knowledge, the problem has never been discussed in literature before. Since the term integrability has various meanings, the notion of integrable boundary conditions has also several definitions. As basic ones we take three definitions, namely, consistency with infinite set of symmetries, consistency with infinite set of conserved quantities, and consistency with the Hamiltonian integrability (or bi-Hamiltonian structure). Comparison of these three kinds of integrable boundary conditions shows that the consistency with the bi-Hamiltonian structure is a very severe restriction. Only very special kind of boundary conditions passes this test. The class of symmetry consistent boundary conditions seems to be relatively larger. As an example we studied the dispersionless Toda system. We found all symmetry compatible boundary conditions of this system and showed that only a subclass of these boundary conditions is com- 
patible with the Hamiltonian formulation of the system. We pointed out that the integrable reductions in the $N$-system of hydrodynamical type of equations are directly related to the integrable boundary conditions of the same systems. Using this property, a method for constructing exact solutions satisfying the integrable boundary conditions is given. We considered also an $N=3$ system. Integrable boundary conditions compatible with symmetries and compatible with the Hamiltonian formulation of this system were found.

\section{ACKNOWLEDGMENTS}

This work was partially supported by the Scientific and Technological Research Council of Turkey (TUBITAK) and Turkish Academy of Sciences (TUBA). One of the authors (I.H.) also thanks Russian Foundation for Basic Research under Grant No. 06-01-92051 KE-a.

${ }^{1}$ B. A. Dubrovin and S. P. Novikov, Sov. Math. Dokl. 27, 665 (1983)

${ }^{2}$ S. P. Tsarev, Sov. Math. Dokl. 31, 488 (1985).

${ }^{3}$ D. J. Benney, Stud. Appl. Math. 52, 45 (1973).

${ }^{4}$ B. A. Kupershmidt and Yu. I. Manin, Funct. Anal. Appl. 11, 188 (1978).

${ }^{5}$ J. Gibbons and S. P. Tsarev, Phys. Lett. A 211, 19 (1996).

${ }^{6}$ B. A. Kupershmidt and Yu. I. Manin, Funct. Anal. Appl. 12, 20 (1978).

${ }^{7}$ J. Gibbons and Y. Kodama, Proceedings of the Singular Limits of Dispersive Waves, edited by N. M. Ercolani, I. R.

Gabitov, C. D. Levermore, and D. Serre (Plenum, New York, 1994).

${ }^{8}$ V. E. Zakharov, Funct. Anal. Appl. 14, 89 (1980).

${ }^{9}$ I. M. Krichever, Funct. Anal. Appl. 22, 200 (1989).

${ }^{10}$ H. Flaschka, M. G. Forest, and D. W. McLaughlin, Commun. Pure Appl. Math. 33, 739 (1980).

${ }^{11}$ E. V. Ferapontov, Am. Math. Soc. Transl. 170, 33 (1995).

${ }^{12}$ M. V. Pavlov, Int. Math. Res. Notices 46987, 43 (2006).

${ }^{13}$ D. B. Fairlie, Physica D 90, 1 (1996).

${ }^{14}$ L. C. Li, Commun. Math. Phys. 203, 573 (1999).

${ }^{15}$ M. Gürses and K. Zheltukhin, J. Math. Phys. 42, 1309 (2001).

${ }^{16}$ B. Gürel, M. Gürses, and I. Habibullin, J. Math. Phys. 36, 6809 (1995).

${ }^{17}$ I. T. Habibullin, Phys. Lett. A 178, 369 (1993).

${ }^{18}$ V. Adler, B. Gürel, M. Gürses, and I. Habibullin, J. Phys. A 30, 3505 (1997).

${ }^{19}$ I. Cherednik, Theor. Math. Phys. 61, 977 (1984).

${ }^{20}$ E. K. Sklyanin, Funkc. Anal. Priloz. 21, 86 (1987).

${ }^{21}$ E. K. Sklyanin, J. Phys. A 21, 2375 (1988).

${ }^{22}$ J. Avan and A. Doikou, Nucl. Phys. B 800, 591 (2008).

${ }^{23}$ A. Doikou, D. Fioravanti, and F. Ravanini, Nucl. Phys. B 790, 465 (2008); e-print arXiv:0706.1515.

${ }^{24}$ D. B. Fairlie and I. A. B. Strachan, Inverse Probl. 12, 885 (1996).

${ }^{25}$ K. Zheltukhin, Phys. Lett. A 297, 402 (2002).

${ }^{26}$ M. A. Semenov-Tyan-Shanskii, Funct. Anal. Appl. 17, 259 (1984).

${ }^{27}$ M. Blaszak, Multi-Hamiltonian Theory of Dynamical Systems (Springer, New York, 1998). 